\title{
Capacity of Diagonally Correlated MIMO Channels
}

\author{
Hüseyin Özcelik \\ Institut für Nachrichtentechnik \\ und Hochfrequenztechnik \\ Technische Universität Wien \\ Vienna, Austria \\ hueseyin.oezcelik@tuwien.ac.at
}

\author{
Claude Oestges \\ Microwave Laboratory \\ Université catholique de Louvain \\ Louvain-la-Neuve, Belgium \\ oestges@emic.ucl.ac.be
}

\begin{abstract}
This paper investigates the case of so-called diagonally correlated MIMO channels. This particular type of correlated channels, which cannot result from the popular Kronecker assumption, are shown to provide higher ergodic capacity than uncorrelated fading channels. Interestingly, such channels present a specific symmetry of the channel matrix element amplitudes: their rows are either cyclically right- or left-shifted versions of each other, so that the absolute value of the channel matrix is circulant. The proposed analysis details exemplary physical scenarios leading to high diagonal correlations, first for the simple case of $2 \times 2$ channels, and then for arbitrary array size $n$. An analytical expression for capacity is also derived. It demonstrates that the ergodic capacity of diagonally correlated channels grows exactly linearly with the number of antennas.
\end{abstract}

\section{INTRODUCTION}

It is well known that the ergodic MIMO capacity grows asymptotically linearly with the minimum number of transmit and receive antennas in a sufficiently rich multipath environment [1], [2]. For Rayleigh-fading channels, it is usually believed that channel correlations always reduce capacity and that therefore independent identically distributed (i.i.d. Rayleigh) channels would yield maximum ergodic capacity. Disregarding bandwidth, the ergodic capacity of a MIMO system with $n$ antennas at both link ends can be expressed as $^{1}$ [1], [2],

$$
\bar{C}_{\mathrm{iid}}=\mathrm{E}_{\mathbf{H}}\left\{\log _{2} \operatorname{det}\left[\mathbf{I}_{n}+\frac{\rho}{n} \mathbf{H} \mathbf{H}^{H}\right]\right\},
$$

where $\mathbf{I}_{n}$ denotes the $n \times n$ identity matrix, $\rho$ the average receive SNR and $\mathbf{H}$ the normalized channel matrix. Rigorously, (1) represents the true capacity (in the Shannon sense) only in i.i.d. scenarios. Still, in correlated channels, it can be seen as a lower bound of capacity, since it represents the mutual information with a non-optimal power allocation (an equal allocation in this case).

However, by introducing a simple metric for the ergodic MIMO capacity, the most surprising result of [3]-[5] was that ergodic capacity is not maximized under i.i.d. fading

\footnotetext{
${ }^{1}$ The following notation will be used throughout this paper: $\mathrm{E}_{x}\{\cdot\}$ stands for the expectation with respect to $x$;

$\log _{2}$ is the logarithm to the base 2 ;

$\operatorname{det}[\cdot]$ is the determinant of a matrix;

$\operatorname{vec}\{\cdot\}$ stacks the elements of a matrix column-wise into a column vector;

$\{\cdot\}^{H}$ denotes Hermitian conjugation;

$\{\cdot\}^{T}$ denotes transposition;

$\odot$ stands for element-wise Schur-Hadamard multiplication.
}

conditions. After exchanging the expectation and the $\log _{2}$ operation (Jensen's inequality) in (1), the proposed metric upper bounds the ergodic mutual information with equal power allocation,

$$
\bar{\kappa}=\log _{2} \mathrm{E}_{\mathbf{H}}\left\{\operatorname{det}\left[\mathbf{I}_{n}+\frac{\rho}{n} \mathbf{H H}^{H}\right]\right\} .
$$

As a beneficial property, the metric allows for analysis of ergodic MIMO capacity as a function of the correlations between different channel matrix elements. Note that the complex correlations between all channel matrix element pairs constitute the full MIMO channel correlation matrix

$$
\mathbf{R}_{\mathbf{H}}=\mathrm{E}\left\{\operatorname{vec}\{\mathbf{H}\} \operatorname{vec}\{\mathbf{H}\}^{H}\right\} .
$$

Interestingly enough, channels with so called diagonal correlations [3], [4] could exhibit higher ergodic capacity than i.i.d. fading channels.

The characterization of such channels is the goal of this paper. To this end, we will introduce, for MIMO $2 \times 2$ systems, exemplary scenarios leading to so-called diagonally correlated channels that exhibit higher ergodic capacity (in the exact Shannon sense) than i.i.d. channels. An analytical expression for the MIMO capacity will be derived. Further, we will extend this approach to arbitrary $n \times n$ diagonally correlated channels.

\section{Diagonally CoRrelated $2 \times 2$ MiMO Channels}

\section{A. Derivation and Modeling}

In case of a $2 \times 2 \mathrm{MIMO}$ channel the channel matrix reads as

$$
\mathbf{H}=\left[\begin{array}{ll}
h_{11} & h_{12} \\
h_{21} & h_{22}
\end{array}\right],
$$

and the full MIMO channel correlation matrix $\mathbf{R}_{\mathbf{H}}$ can be expressed as

$$
\left[\begin{array}{llll}
\mathrm{E}\left\{h_{11} h_{11}^{*}\right\} & \mathrm{E}\left\{h_{11} h_{21}^{*}\right\} & \mathrm{E}\left\{h_{11} h_{12}^{*}\right\} & \mathrm{E}\left\{h_{11} h_{22}^{*}\right\} \\
\mathrm{E}\left\{h_{21} h_{11}^{*}\right\} & \mathrm{E}\left\{h_{21} h_{21}^{*}\right\} & \mathrm{E}\left\{h_{21} h_{12}^{*}\right\} & \mathrm{E}\left\{h_{21} h_{22}^{*}\right\} \\
\mathrm{E}\left\{h_{12} h_{11}^{*}\right\} & \mathrm{E}\left\{h_{12} h_{21}^{*}\right\} & \mathrm{E}\left\{h_{12} h_{12}^{*}\right\} & \mathrm{E}\left\{h_{12} h_{22}^{*}\right\} \\
\mathrm{E}\left\{h_{22} h_{11}^{*}\right\} & \mathrm{E}\left\{h_{22} h_{21}^{*}\right\} & \mathrm{E}\left\{h_{22} h_{12}^{*}\right\} & \mathrm{E}\left\{h_{22} h_{22}^{*}\right\}
\end{array}\right] .
$$

The entries of the main diagonal of $\mathbf{R}_{\mathbf{H}}$ represent the 
antenna powers ${ }^{2}, \mathrm{E}\left\{h_{i j} h_{l j}^{*}\right\}$ with $i \neq j \neq l$ are the receive antenna correlations, and $\mathrm{E}\left\{h_{i j} h_{i l}^{*}\right\}$, again with $i \neq j \neq l$, are the transmit antenna correlations, respectively. Thus, following [3], [4], we will call the remaining matrix entries diagonal correlations.

According to [5], the mutual information metric given by (2) can be maximized for a $2 \times 2$ MIMO Rayleigh fading channel with equal power allocation across the antennas if the absolute value of the full channel correlation matrix, $\left|\mathbf{R}_{\mathbf{H}}\right|$, fulfills

$$
\left|\mathbf{R}_{\mathbf{H}}\right|=\left[\begin{array}{llll}
1 & 0 & 0 & 1 \\
0 & 1 & 1 & 0 \\
0 & 1 & 1 & 0 \\
1 & 0 & 0 & 1
\end{array}\right],
$$

i.e. if the antenna amplitude correlations are zero, but the magnitudes of the diagonal correlations are equal to unity. We call the type of channels outlined by (5) a $2 \times 2$ diagonally correlated channel. Thus, the amplitudes of its matrix elements read as

$$
|\mathbf{H}|=\left[\begin{array}{ll}
\left|h_{11}\right| & \left|h_{12}\right| \\
\left|h_{12}\right| & \left|h_{11}\right|
\end{array}\right] .
$$

An exemplary scenario leading to a diagonally correlated $2 \times 2$ MIMO channel is illustrated in Fig. 1. This scenario exhibits two nearly equally-powered, independent Rayleighfading multipath components with orthogonal directions-ofdeparture (DoDs) and directions-of-arrival (DoAs). If a single DoD couples into a single DoA and vice versa, then the diagonally correlated channel results [6]. Note that 'orthogonal' in this sense does not imply right angles, but orthogonality of the corresponding steering/response vectors.

Using the formalism of the finite scatterer model [7] or the approach presented by [8], the just proposed exemplary diagonally correlated channel $\mathbf{H}$ can be expressed as

$$
\mathbf{H}=\mathbf{A}_{\mathrm{Rx}}(\mathbf{D} \odot \mathbf{G}) \mathbf{A}_{\mathrm{Tx}}^{T} .
$$

Here, $\mathbf{G}$ is a $2 \times 2$ i.i.d. fading matrix with zero-mean, complex circularly symmetric Gaussian entries. The two columns of the unitary matrix $\mathbf{A}_{\mathrm{Tx}}\left(\mathbf{A}_{\mathrm{Rx}}\right)$ constitute orthogonal steering (response) vectors of DoDs (DoAs). The $2 \times 2$ coupling matrix D is either a diagonal or anti-diagonal matrix with identical entries, producing the equally-powered, one-to-one coupling between DoDs and DoAs. Almost identical entries of the coupling matrix D work fine as well. We emphasize that a diagonally correlated MIMO channels may result from the virtual channel representation [8] with a diagonal (or antidiagonal) coupling matrix ${ }^{3}$.

Yet, it is important to observe that diagonally correlated channels cannot result from the application of the popular Kro-

\footnotetext{
${ }^{2} \mathrm{We}$ assume in this paper that all antenna powers are equal, so the main diagonal of $\mathbf{R}_{\mathbf{H}}$ consists of equal entries.

${ }^{3}$ MIMO channels that provide higher ergodic capacity than i.i.d. channels also result from the Weichselberger model [9]

$$
\mathbf{H}=\mathbf{U}_{\mathrm{Rx}}(\mathbf{D} \odot \mathbf{G}) \mathbf{U}_{\mathrm{Tx}}^{T},
$$
}

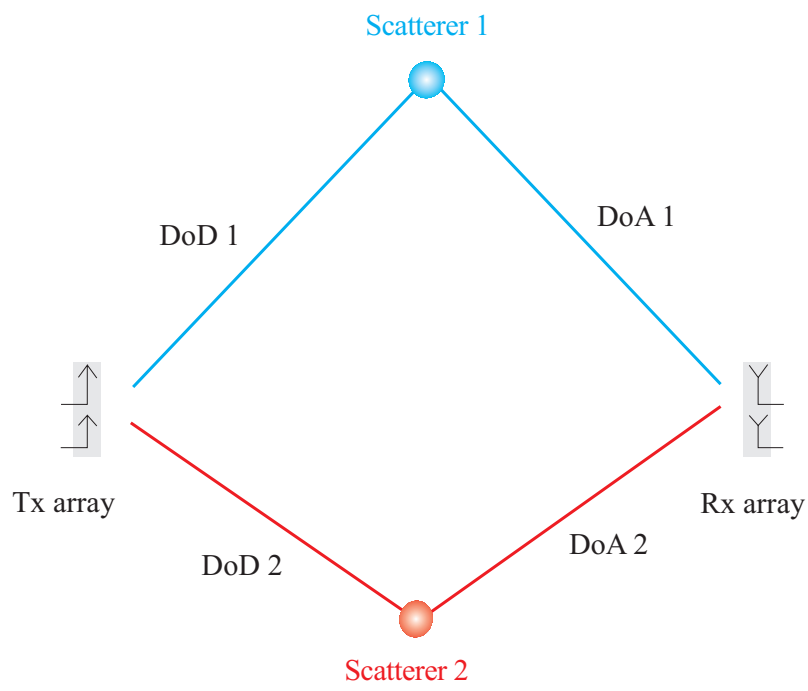

Fig. 1. Illustration of an exemplary scenario leading to a $2 \times 2$ diagonally correlated channel.

necker model [10]-[13]. At this stage, it should be reminded that the Kronecker model is based on independence between DoDs and DoAs and therefore couples any DoD into all DoAs and vice versa (see [4], [14] for a summary of the assumptions of the Kronecker model). This is exactly the opposite of the diagonally correlated channel illustrated in Fig. 1.

\section{B. Capacity}

A closer look at (7) reveals the distribution of the singular values of $\mathbf{H}$. The columns of the unitary matrix $\mathbf{A}_{\mathrm{Tx}}\left(\mathbf{A}_{\mathrm{Rx}}\right)$ constitute the right (left) singular vectors, except a constant phase term. The magnitudes of the singular values of $\mathbf{H}$, $\left|s_{i}\right|$, are given by the magnitudes of the complex-Gaussian distributed, non-zero elements of $\mathbf{D} \odot \mathbf{G}$. Hence, $\left|s_{i}\right|$ follows a Rayleigh distribution. As a consequence, the eigenvalues $\lambda_{i}=\left|s_{i}\right|^{2}$ of the Gramian $\mathbf{H H}^{H}$ are Chi-squared distributed with two degrees of freedom.

Before calculating the ergodic capacity, the channel has to be normalized properly. Its normalization to unity average power of each channel matrix coefficient leads to a Rayleigh parameter for $\left|s_{i}\right|$ of unity, too.

The ergodic capacity of a $2 \times 2$ diagonally correlated channel is exactly given by [15]

again with a diagonal (or anti-diagonal) coupling matrix. Here, $\mathbf{U}_{\mathrm{Tx}}$ and $\mathbf{U}_{\mathrm{Rx}}$ denote the unitary eigenbasis at the transmitter and receiver, i.e. the eigenvectors of the transmit (receive) correlation matrix constitute the columns of $\mathbf{U}_{\mathrm{Tx}}\left(\mathbf{U}_{\mathrm{Rx}}\right)$. In contrast to (7), channels modeled by (3) do not exhibit diagonal correlations only. However, space does not allow a more detailed examination here. 


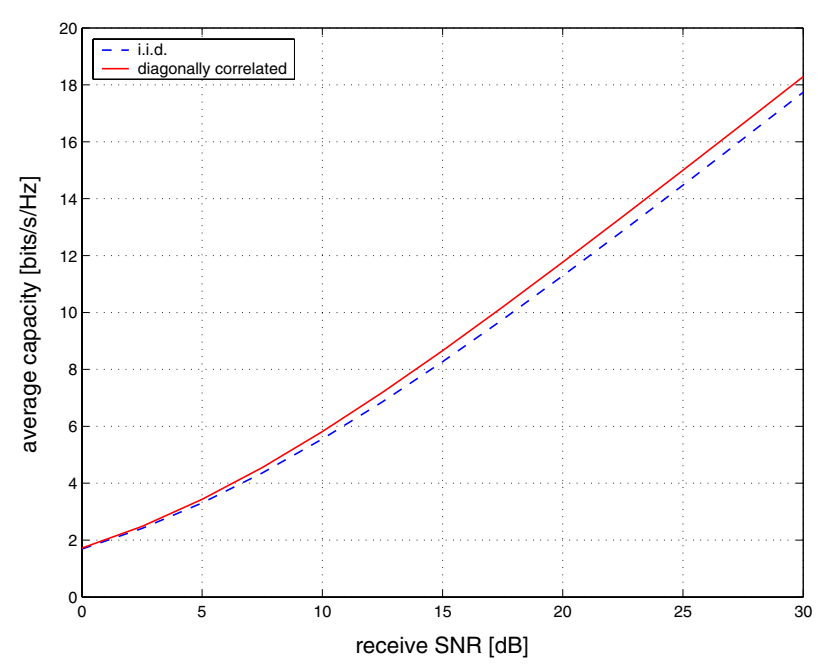

Fig. 2. Average capacity of a $2 \times 2$ i.i.d. and diagonally correlated channel as a function of the average receive SNR.

$$
\begin{aligned}
\bar{C} & =\mathrm{E}_{x}\left\{\sum_{k=1}^{2} \log _{2}\left(1+\frac{\rho}{2} x^{2}\right)\right\} \\
& =2 \cdot \mathrm{E}_{x}\left\{\log _{2}\left(1+\frac{\rho}{2} x^{2}\right)\right\} \\
& =2 \int_{0}^{\infty} \log _{2}\left(1+\frac{\rho}{2} x^{2}\right) x e^{-\frac{x^{2}}{2}} d x \\
& =2 \log _{2}(e) e^{\frac{1}{\rho}} \mathrm{E}_{1}\left(\frac{1}{\rho}\right),
\end{aligned}
$$

where $x$ denotes a random realization of the identically distributed eigenvalues $\lambda_{i}$, and $\mathrm{E}_{1}(z)$ is the En-Function [16] for $\mathrm{n}=1$ that satisfies $\mathrm{E}_{1}(z)=\int_{1}^{\infty} \frac{e^{-t z}}{t} d t$. For the special case of $\mathrm{n}=1$, the En-Function can also be expressed in terms of the Exponential Integral Function $\operatorname{Ei}(z)$, i.e. $\mathrm{E}_{1}(z)=-\operatorname{Ei}(-z)$.

Close scrutiny of $(8)$ reveals that $2 \times 2$ diagonally correlated channels exhibit a higher ergodic capacity than the corresponding i.i.d. channels. Monte-Carlo simulations with $5 \cdot 10^{5}$ realizations for each SNR value were performed in order to show that this is indeed the case.

Fig. 2 plots the resulting average $4 \times 2$ MIMO capacity of an i.i.d. and a diagonally correlated channel as a function of the receive SNR $\rho$. Evidently, the diagonally correlated channel exhibits a higher ergodic capacity. (Of course, both channels carry the same average power.) The difference is small for $2 \times 2$ systems, but it discusses the general statement that i.i.d. gives maximum ergodic capacity.

Further, we compared the capacity cdf of a diagonally correlated channel with the one of an i.i.d. channel at an SNRlevel of $20 \mathrm{~dB}$. Fig. 3 shows the outcome. Despite the fact that the diagonally correlated channel (solid line) provides a higher average capacity than the i.i.d. channel (dashed line),

\footnotetext{
${ }^{4}$ We use the term ergodic only with respect to random variables. When dealing with realizations of a random variable, the expected value can be estimated by the arithmetic mean or the average.
}

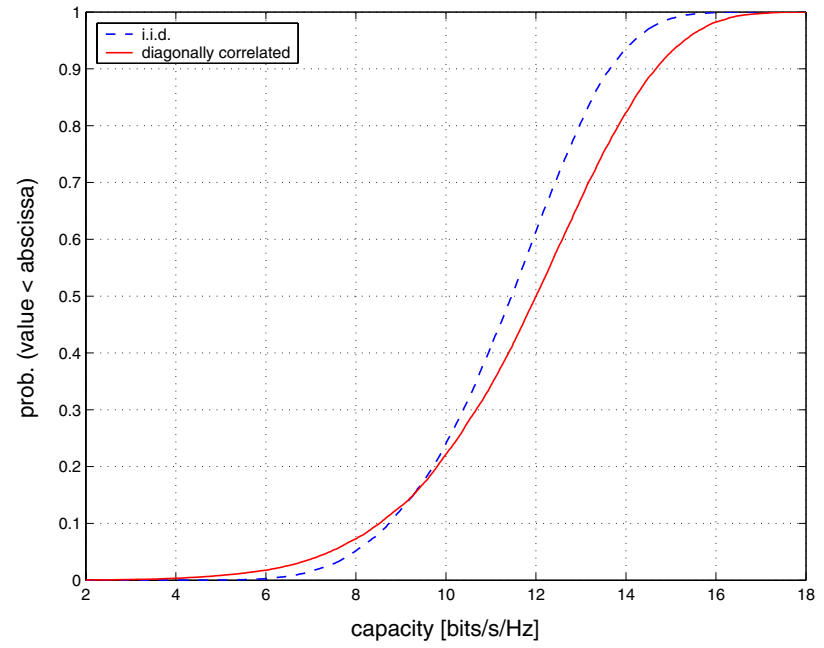

Fig. 3. Capacity cdf of a $2 \times 2$ i.i.d. and diagonally correlated channel at $20 \mathrm{~dB}$ receive SNR.

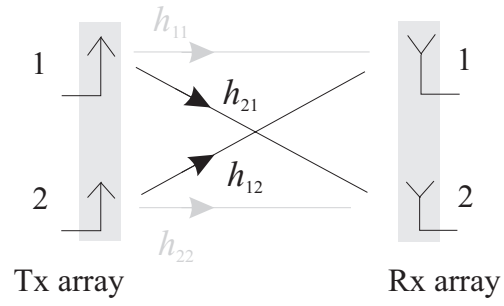

Fig. 4. The $2 \times 2$ diagonally correlated channel: fading pair $\left|h_{21}\right|-\left|h_{12}\right|$.

we observe that the slope of its capacity cdf is flatter. Its outage capacity is lower for outage levels higher than approximately $15 \%$.

\section{Fading Behavior}

As already mentioned before, for diagonally correlated channels $\left|h_{11}\right|=\left|h_{22}\right|$ and $\left|h_{12}\right|=\left|h_{21}\right|$. As a consequence, the channel amplitudes fade in tandem, either in pairs of $\left|h_{11}\right|-\left|h_{22}\right|$ (Fig. 5) or $\left|h_{12}\right|-\left|h_{21}\right|$ (Fig. 4), respectively. But these pairs fade Rayleigh independently of each other.

By contrast, in case of i.i.d. Rayleigh-fading, each single channel matrix entry $h_{i j}$ fades independently (according to a Rayleigh distribution).

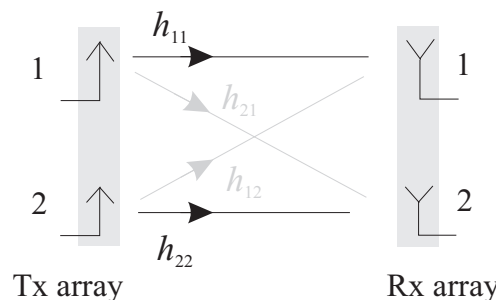

Fig. 5. The $2 \times 2$ diagonally correlated channel: fading pair $\left|h_{11}\right|-\left|h_{22}\right|$. 


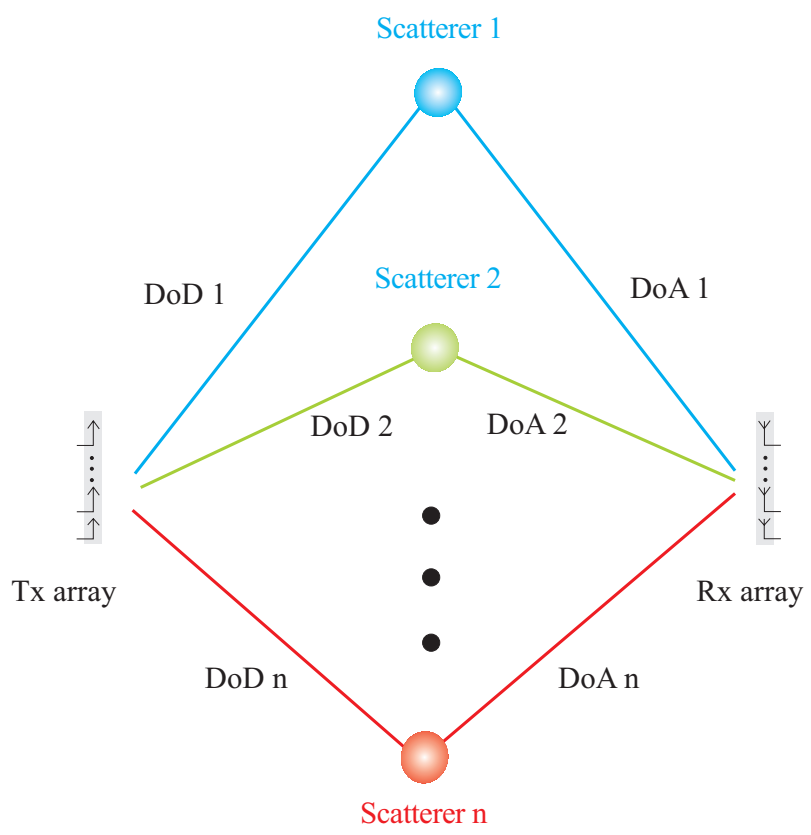

Fig. 6. Illustration of an exemplary $n \times n$ diagonally correlated channel: A channel with $n$ orthogonal DoDs and $n$ orthogonal DoAs such that each single DoD couples with equal power into a single DoA and vice versa.

An intuitively appealing reason for the capacity increase is as follows. Each of the independent fading pairs of a diagonally correlated channel provides perfectly separated virtual sub-channels on their own. This improved separation of the sub-channels results in a higher ergodic capacity. But, since the degrees of freedom reduce from four to two, diversity in diagonally correlated channels is lower than for i.i.d., leading to a flatter slope of the cdf, i.e. lower outage capacity for low outage levels.

\section{Diagonally Correlated $n \times n$ MIMO Channels}

The concept of the proposed $2 \times 2$ diagonally correlated channel can be generalized to $n \times n$ systems as follows. Consider a symmetric $n \times n$ MIMO channel with $n$ orthogonal DoDs and $n$ orthogonal DoAs. We call such a channel diagonally correlated if each single $\mathrm{DoD}$ couples into a single DoA and vice versa. This is illustrated in Fig. 6.

The analytical modeling remains identical and follows (7). Of course, the dimensions of the unitary steering and response matrices $\mathbf{A}_{\mathrm{Tx}}$ and $\mathbf{A}_{\mathrm{Rx}}$, the coupling matrix, and the random fading matrix $\mathbf{G}$ must change to $n \times n$. Further, $\mathbf{D}$ is a sparse matrix with only one entry in each row and column, producing the one-to-one coupling between DoDs and DoAs. The entries of $\mathbf{D}$ have to be identical. The actual shape of $\mathbf{D}$ determines the appearance of the channel matrix and therefore the full MIMO channel correlation matrix.

It is interesting to note that the amplitudes of the corresponding channel matrix entries exhibit a specific symmetry. The rows of these amplitudes have to be cyclically right- or left-shifted versions of each other. As an example for $3 \times 3$

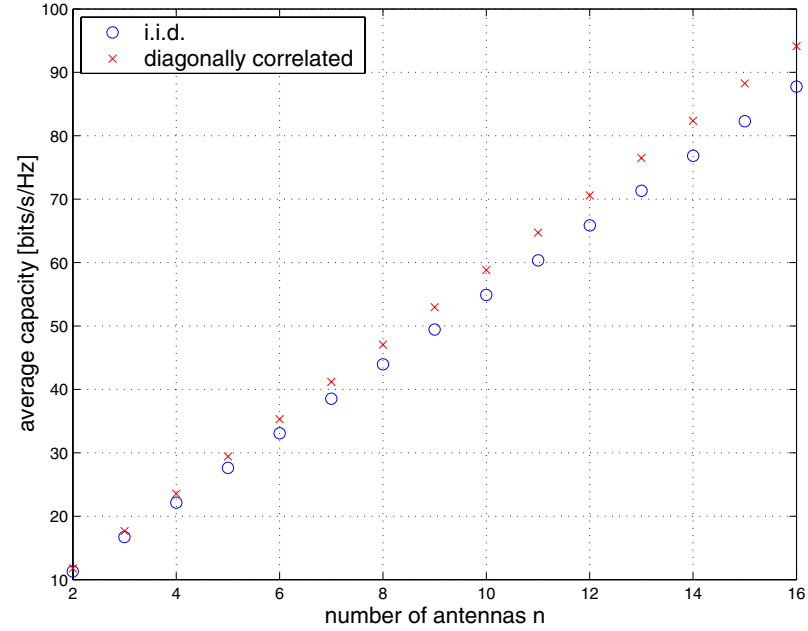

Fig. 7. Average capacity of an i.i.d. and diagonally correlated channel at $20 \mathrm{~dB}$ receive SNR as a function of the antenna number.

MIMO, the amplitudes of the channel matrix may result, for a given choice of $\mathbf{D}$, in

$$
|\mathbf{H}|=\left[\begin{array}{lll}
\left|h_{11}\right| & \left|h_{12}\right| & \left|h_{13}\right| \\
\left|h_{12}\right| & \left|h_{13}\right| & \left|h_{11}\right| \\
\left|h_{13}\right| & \left|h_{11}\right| & \left|h_{12}\right|
\end{array}\right],
$$

or

$$
|\mathbf{H}|=\left[\begin{array}{lll}
\left|h_{11}\right| & \left|h_{12}\right| & \left|h_{13}\right| \\
\left|h_{13}\right| & \left|h_{11}\right| & \left|h_{12}\right| \\
\left|h_{12}\right| & \left|h_{13}\right| & \left|h_{11}\right|
\end{array}\right] .
$$

However, the eigenvalues $\lambda_{i}=\left|s_{i}\right|^{2}$ of the Gramian $\mathbf{H H}^{H}$ are still independently identical Chi-squared distributed with two degrees of freedom, independent of the actual shape of D. If the channel is normalized to unity average power of each channel matrix coefficient, the variance of the Rayleigh distributed $\left|s_{i}\right|$ increases linearly with the number of antennas. For $n$ antennas at both link ends, it equals $\frac{n}{2}$. Hence, the ergodic capacity of an $n \times n$ diagonal channel with identically distributed eigenvalues $\lambda_{i}$ of its Gramian is exactly given by [15]

$$
\begin{aligned}
\bar{C} & =\mathrm{E}_{x}\left\{\sum_{k=1}^{n} \log _{2}\left(1+\frac{\rho}{n} x^{2}\right)\right\} \\
& =n \log _{2}(e) e^{\frac{1}{\rho}} \mathrm{E}_{1}\left(\frac{1}{\rho}\right),
\end{aligned}
$$

where $x$ denotes, again, a random realization of the eigenvalues $\lambda_{i}$.

Note that in the above expression the capacity grows exactly linearly with the number of antennas while the capacity of i.i.d. channels grows in $n$ only asymptotically.

Fig. 7 shows the average MIMO capacity of an i.i.d. channel (circles) and a diagonally correlated channel (crosses) as a function of the antenna number $n$, at a receive SNR of 
20dB. It can be seen that the absolute difference in average MIMO capacity increases with increasing antenna number. Furthermore, the relative capacity increase also grows with $n$ from $3.5 \%(n=2)$ to almost $7 \%(n=16)$.

\section{CONCLUSIONS}

This paper has shown that so-called diagonally correlated MIMO channels have the remarkable property of providing higher ergodic capacity than i.i.d. channels. The difference in ergodic capacity increases with increasing antenna number. The study also proposes exemplary scenarios that allow for such behavior to take place. Diagonally correlated channels can be modeled by the virtual channel representation with a sparse coupling matrix that has only one entry in each of its rows and columns. An analytical formula for the ergodic capacity of symmetric MIMO channels with arbitrary antenna number $n$ and an intuitively appealing reason for the capacity increase have been detailed.

\section{ACKNOWLEDGMENTS}

Part of this work was supported by the EuropeanCommission-funded Network of Excellence NEWCOM.

The authors wish to thank Prof. Ernst Bonek (Technische Universität Wien, Austria) for his most helpful comments.

\section{REFERENCES}

[1] I.E. Telatar, 'Capacity of Multi-Antenna Gaussian Channels', in Technical Memorandum, Bell Laboratories, Lucent Technologies, vol. 1, Orlando, FL, Oct. 1998, pp. 394-398, published in European Transactions on Telecommunications, vol. 10, no. 6, pp. 585-595, Nov./Dec. 1999.

[2] G.J. Foschini, and M.J. Gans, 'On Limits of Wireless Communications in Fading Environments when Using Multiple Antennas', in Wireless Personal Communication, vol. 6, 1998, pp. 311-335.

[3] C. Oestges, B. Clerckx, D. Vanhoenacker-Janvier, and A.J. Paulraj, 'Impact of Diagonal Correlations on MIMO Capacity: Application to Geometrical Scattering Models', in Proc. 58th IEEE Vehicular Technology Conference Fall (VTC-F '03), vol. 1, Orlando, FL, Oct. 2003, pp. 394-398.
[4] C. Oestges, B. Clerckx, D. Vanhoenacker-Janvier, and A.J. Paulraj, 'Impact of Fading Correlations on MIMO Communication Systems in Geometry-Based Statistical Channel Models', IEEE Transactions on Wireless Communications, vol. 40, no. 3, 2005.

[5] C. Oestges and A.J. Paulraj, 'Beneficial Impact of Channel Correlations on MIMO Capacity', Electronic Letters, vol. 40, no. 10, 2004, pp. 606607.

[6] H. Özcelik, and E. Bonek, 'Diagonal-Correlation Channels: Better than i.i.d.?', COST 273 TD (04) 13, June 2004, Gothenburg, Sweden.

[7] A.G. Burr, 'Capacity Bounds and Estimates for the Finite Scatterers MIMO Wireless Channel', IEEE Journal on Selected Areas in Commun. vol. 21, no. 5, 2003, pp. 812-818.

[8] A.M. Sayeed, 'Deconstructing Multi-antenna Fading Channels', IEEE Transactions on Signal Processing, vol. 20, no. 10, 2002, pp. 25632579.

[9] W. Weichselberger, M. Herdin, H. Ozcelik, and E. Bonek, 'A Stochastic MIMO Channel Model with Joint Correlation of Both Link Ends', IEEE Transactions on Wireless Communications, in press.

[10] Chen-Nee Chuah, J.M. Kahn, and D.N.C. Tse, 'Capacity of MultiAntenna Array Systems in Indoor Wireless Environment', IEEE Global Telecommunications Conference, vol. 4, Sidney, Australia, 1998, pp. 1894-1899.

[11] D. Chizhik, F. Rashid-Farrokhi, J. Ling, and A. Lozano, 'Effect of Antenna Separation on the Capacity of BLAST in Correlated Channels', IEEE Communications Letters, vol. 4, no. 11, Nov. 2000, pp. 337-339.

[12] Da-Shan Shiu, G.J. Foschini, M.J. Gans, and J.M. Kahn, 'Fading Correlation and Its Effect on the Capacity of Multielement Antenna Systems', IEEE Transactions on Communications, vol. 48, no. 3, Mar. 2000, pp. 502-513.

[13] J.P. Kermoal, L. Schumacher, K.I. Pedersen, P.E. Mogensen, and F. Frederiksen, 'A Stochastic MIMO Radio Channel Model with Experimental Validation', IEEE Journal on Selected Areas in Communications, vol. 20, no. 6, Aug. 2002, pp. 1211-1226.

[14] H. Ozcelik, M. Herdin, W. Weichselberger, J. Wallace, and E. Bonek, 'Deficiencies of Kronecker MIMO Radio Channel Model', Electronic Letters, vol. 39, no. 16-7, 2003, pp. 1209-1210.

[15] H. Özcelik, and C. Oestges, 'Some Remarkable Properties of Diagonally Correlated MIMO Channels', Electronic Letters, submitted.

[16] M. Abramowitz, and I.A. Stegun (Eds.), 'Exponential Integral and Related Functions', New York: Dover, 1972, Chapter 5 in Handbook of Mathematical Functions with Formulas, Graphs, and Mathematical Tables. 